\title{
DERIVATION OF THE AMPLITUDE EQUATIONS OF ACOUSTIC MODES \\ OF AN UNSTABLE SEMI-INFINITE POLYTROPE*
}

\author{
(Invited Review) \\ JEAN - PIERRE POYET \\ Observatoire du Pic-du-Midi et de Toulouse, 14 Avenue Edouard Belin, 31400 Toulouse, France
}

\begin{abstract}
The coupling of non-radial pulsations and convection is studied on a simple example: an unstable semi-infinite polytrope. An expansion is proposed and the equations that should describe correctly the pulsations are isolated. The additional complications of the real solar case are discussed at the end.
\end{abstract}

\section{Introduction}

The purpose of this study is to try to establish what kinds of equations are obeyed by the solar pulsations and to illustrate the method by a simple example. One of the main causes of the awkwardness of the solar problem is the presence of a convective zone, given the lack of any theory of convection so far. Moreover, the fuzziness and the scarcity of the observations make it difficult to test even a simple empirical theory such as the mixing length theory, as is well illustrated by Gough and Weiss (1976). Fortunately, the increasing accuracy of present measurements is beginning to give information about the evolution of the amplitude of individual pulsation modes (see Grec et al., 1983) and their correlation time (Grec et al., 1980). As a consequence, it is time to try to write precise equations and to settle certain ambiguities about the present state of the treatment of the interaction of convection and pulsation in a star.

In the case of a star in purely radial pulsation, it is clear how to separate the pulsating motion from the convective one: a suitable time average generally suffices (Gough, 1976; Unno, 1967, 1977; Poyet and Spiegel, 1979). In the case of non-radial pulsations the situation is less clear and the problem is really well-defined only when a separation of scale is assumed: the convective motions being supposed to arise on scales much smaller than the pulsating motions. One can then perform a two-scale development of the problem and average over the small convective scales (Unno et al., 1979). In this limit only, the concept of turbulent viscosity, introduced to model the effects of the convection zone, can be justified physically. However this is not the case in the Sun since it is generally believed by people studying its convective zone that some cells of about the size of the convection zone $(\approx 200000 \mathrm{~km})$ must be present and there are theoretical reasons which might lead one to believe in the presence of even larger scales (in linear theory, it is found, in Boussinesq convection between plane parallel layers, that the

\footnotetext{
* Proceedings of the 66th IAU Colloquium: Problems in Solar and Stellar Oscillations, held at the Crimean Astrophysical Observatory, U.S.S.R., 1-5 September, 1981.
} 
convective instability arises first at zero wavenumber for boundary conditions with fixed flux; see Hurle et al., 1967). So theoretically, it should be assumed that convection covers scales from the granules $(l \approx 1000$, where $l$ is the usual index of the spherical harmonics), or even from the subgranular scales that are likely to be turbulent, to the solar circumference, and consequently that convective and pulsating scales in the Sun are comparable. Thus there is no easy physical way to separate them and an arbitrary definition must be adopted. Given any type of well-defined linear problem, either of the type of the simple polytropic example that will be treated later or coming from a numerical program, the modes will be classified according to their time-dependence $e^{i \sigma t}$, where, in general, $\sigma=\sigma_{R}+i \sigma_{I}$ with $\sigma_{R}$ and $\sigma_{I}$ real. If $\left|\sigma_{I}\right| \ll \mid \sigma_{R}$, the mode will be considered as a pulsating one. If this is not true, the modes will be treated as convective ones (overstable if $\left|\sigma_{R}\right|$ is different from zero). As an illustration, for a superadiabatic polytrope (unstable to convection) the $f$ and $p$ modes will be considered as pulsating modes, while the $g^{-}$and toroidal modes (with zero frequencies) will be considered as belonging to the convective modes.

Another outstanding problem of the subject is the perturbation of the convective flux. It is generally ignored because it involves a time-dependent theory of convection. In the solar case, one can imagine trying to tackle the problem because, despite the non-linearity due to the convection, the pulsations are of such small amplitudes that they must behave almost linearly in some sense. The question is then to attempt to isolate this almost linear problem theoretically and use all of its nice linear properties to understand the pulsationconvection coupling. When observers interpret the peaks in their spectra as normal modes of the Sun, they do nothing else but assume the existence of these linear properties, despite the messy couplings with the convection. Given these facts, there are two ways one can think of, to start the problem. On one hand, some convective terms in the mean equations that one wishes to start with could be kept. Then one is faced with the rather difficult problem of an unknown mean state to calculate further. This would generally force one to close the convection equations (via the mixing-length theory for instance) already in the mean state. This procedure has two disadvantages: the first one is that one has to perturb a bad theory (the mixing length theory, for example) to calculate even the linear problem and that there is no unique way of doing it; the second one is that the closure is on the mean state of the convection and it is well known, in usual turbulence theory, that such theories give results which are rather worse than those obtained by trying to close on higher order correlations. On the other hand, it seems more natural to perturb exact equations and then to try to close the problem. This is the method adopted in what follows, where the exact equations for the convection are kept and then perturbed. A very simple example will be treated, embodying, in our opinion, the conceptual difficulties of the problem. The additional difficulties of the real solar problem will be commented on at the end.

Before starting the calculation for an unstable polytrope, it is useful to review the motivations for doing such a non-linear calculation. One of the issues about the Sun, that should be settled by a good non-linear theory, is the nature of the excitation of the pulsating modes. It has sometimes been believed, after the unsuccessful attempt by 
Goldreich and Keeley $(1977 \mathrm{a}, \mathrm{b})$ to excite the modes stochastically by turbulent convection, that the $\kappa$-mechanism was at work, as confirmed by the calculation of Ando and Osaki (1975a, b). However the uncertainties in the method used by Goldreich and Keeley; the fact that a more accurate treatment of radiative transfer stabilizes the modes found unstable by Ando and Osaki (see the article by Christensen-Dalsgaard and Frandsen, 1983); and the behaviour of the amplitude of the modes identified in the South Pole experiment (see the article by Grec et al., 1983) induce one to think that indeed the convection zone is exciting the modes. Two things must be explained by the proponents of the $\kappa$-mechanism as an excitation process: why doesn't the Sun pulsate with the amplitude of a Cepheid and, more importantly, why is there a lifetime of the modes of two days in Fossat's results (one would rather expect a stationnary solution with the $\kappa$-mechanism)? However it is not excluded that some contribution to the excitation of the pulsation comes from the $\kappa$-mechanism and, later on, the inclusion of such an effect in a realistic calculation will be discussed. In any case, a more realistic treatment of the convection is needed and we shall concentrate on this problem here.

\section{The Unstable Polytrope}

We consider an adiabatic problem and take as origin the surface of the star $\left(z=z_{s}\right)$ with a $z$-axis oriented positively inward. We suppose that there is no viscosity or thermal conductivity and that $\gamma$, the usual ratio of specific heats, is constant. We neglect any radiative effect and suppose that the gravity is constant. The fluid hydrodynamical equations for such a problem are

$$
\begin{aligned}
& \frac{\partial \rho}{\partial t}+\nabla \cdot(\rho \mathbf{v})=0 \\
& \rho \frac{\mathrm{d} v_{i}}{\mathrm{~d} t}=-\frac{\partial p}{\partial x_{i}}+\rho g \delta_{i 3}, \quad i=1,2,3 \\
& \frac{d p}{\mathrm{~d} t}=c^{2} \frac{\mathrm{d} \rho}{\mathrm{d} t}=-c^{2} \rho \nabla \cdot \mathbf{v}=-\gamma p \nabla \cdot \mathbf{v} \\
& c^{2}=\gamma \frac{p}{\rho}
\end{aligned}
$$

where $\rho, \mathbf{v}$, and $p$ are the density, velocity, and pressure in the medium. Indices 1 and 2 for $i$ are for the horizontal velocity and 3 is for the vertical velocity. An infinite plane paralel layer is considered. One starts by defining a polytropic state when $\mathbf{v}=0$. A reference layer, $z=z_{0}$, is chosen for this polytrope such that at $z=z_{0}, T_{0}=\Theta$, and $p_{0}=P$, where the index zero designates polytropic variables. It follows that

$$
T_{0}=\beta z,
$$




$$
\begin{aligned}
& p_{0}=P\left(\frac{z}{z_{0}}\right)^{m+1}=K z^{m+1}, \\
& \rho_{0}=\frac{K}{R \beta} z^{m}, \\
& K=\frac{P}{z_{0}^{m+1}}, \\
& m=\frac{g}{R \beta}-1, \\
& c_{0}^{2}=\gamma \frac{p_{0}}{\rho_{0}}=\gamma R \beta z=\frac{\gamma g}{m+1} z,
\end{aligned}
$$

where $\beta$ is the constant static temperature gradient, $R$ the constant of the perfect gases, $m$ the polytropic index, and $c_{0}$ the sound speed for the polytrope. A polytrope of thickness $d$ is considered, so that

$$
z_{s}<z<z_{s}+d
$$

and the thickness $d$, when it is finite, can be taken for $z_{0}$ for example. The case of the infinite polytrope that is treated later will be given simply by taking $z_{s}=0$ and $d \rightarrow \infty$. The equations are nondimensionalized by choosing the length $z_{0}$ and an arbitrary time $\tau$ as units.

It is now convenient to introduce potential variables. Potential pressure $\pi$ and temperature $\theta$ are defined by

$$
\begin{aligned}
& \pi=\left(\frac{p}{P}\right)^{R / c_{p}}, \\
& T=\Theta \pi \theta .
\end{aligned}
$$

Remembering that

$$
\frac{R}{c_{p}}=\frac{\gamma-1}{\gamma}
$$

using $\theta$ and $\pi$ instead of $T$ and $p$ and eliminating $\rho$ from the equations via the equation of state of perfect gases

$$
p=\rho R T,
$$

one obtains the following equations:

$$
\lambda_{a} M^{2} \frac{\mathrm{d} \mathbf{v}}{\mathrm{d} t}=-\theta \nabla \pi+\lambda_{a} \delta_{i 3},
$$




$$
\begin{aligned}
& \frac{\mathrm{d} \pi}{\mathrm{d} t}=-\pi(\gamma-1) \nabla \cdot \mathbf{v}, \\
& \frac{\mathrm{d} \theta}{\mathrm{d} t}=0,
\end{aligned}
$$

with

$$
\begin{aligned}
\lambda_{a} & =\frac{g}{c_{p} \beta}, \\
M^{2} & =\frac{\left(z_{0} / \tau\right)^{2}}{g z_{0}},
\end{aligned}
$$

where $\lambda_{a}$ is the ratio of the adiabatic and static temperature gradients and $M^{2}$ can be interpreted as the ratio of a typical velocity to a free-fall velocity and is analogous to a Mach number squared. Such equations have been used by Ogura and Phillips (1962) to derive the anelastic approximation from the convection equations in the atmosphere. That approximation is obtained by an asymptotic expansion to $O\left(M^{2}\right)$ of the preceding equations. The main property of the anelastic equations is that the acoustic waves have been filtered out and that they should be an accurate representation of purely convective phenomena. Consequently, it will be interesting to trace their role, in the final equations derived later, by considering the $M^{2}$ parameter. (For the derivation of this approximation in a realistic stellar case, see Gough (1969).)

One might wonder, at this stage, about the usefulness of potential variables. On top of the fact that they simplify somewhat the initial equations by giving only quadratic non-linearities, it will be seen that the linear problem for the complete velocity and thermodynamic fields makes them the natural variables to utilize.

The polytropic state can be rewritten with the new potential variables, taking into account the units of time and length $\tau$ and $z_{0}$, as

$$
\begin{aligned}
& \pi_{0}=z^{m+1-[(m+1) / \gamma]}, \\
& 0_{0}=z^{[(m+1) / ;]-m}, \\
& c_{0}^{2}=\frac{\gamma-1}{\lambda_{a} M^{2}} z .
\end{aligned}
$$

In terms of these variables, the hydrostatic mean state equation becomes

$$
\theta_{0} \frac{\mathrm{d} \pi_{0}}{\mathrm{~d} z}=\lambda_{a}
$$

A quantity $f(x, y, z, t)$ will be expressed as

$$
f(x, y, z, t)=f_{0}(z)+f^{\prime}(x, y, z, t)
$$


where $f_{0}$ describes the polytropic state. From Equations (2.16), (2.17), and (2.18), one can get equations for the primed quantities,

$$
\begin{aligned}
& \frac{\partial \mathbf{v}^{\prime}}{\partial t}+\frac{\theta_{0}}{\lambda_{a} M^{2}} \nabla \pi^{\prime}+\frac{1}{\lambda_{a} M^{2}} \frac{\mathrm{d} \pi_{0}}{\mathrm{~d}_{z}} \delta_{i 3} \theta^{\prime}=-\mathbf{v}^{\prime} \cdot \nabla \mathbf{v}^{\prime}-\frac{\theta^{\prime} \nabla \pi^{\prime}}{\lambda_{a} M^{2}}, \\
& \frac{\partial \pi^{\prime}}{\partial t}+\pi_{0}(\gamma-1) \nabla \cdot \mathbf{v}^{\prime}+\frac{\mathrm{d} \pi_{0}}{\mathrm{~d} z} w^{\prime}=-\mathbf{v}^{\prime} \cdot \nabla \pi^{\prime}-(\gamma-1) \pi^{\prime} \nabla \cdot \mathbf{v}^{\prime}, \\
& \frac{\partial \theta^{\prime}}{\partial t}+\frac{\mathrm{d} \theta_{0}}{\mathrm{~d} z} w^{\prime}=-\mathbf{v}^{\prime} \cdot \nabla \theta^{\prime},
\end{aligned}
$$

where $w^{\prime}$ is the vertical component of the velocity. The left-hand side of $(2.26),(2.27)$, and (2.28) constitutes the linear problem. By Fourier-transforming it horizontally, one obtains

$$
\begin{aligned}
& \frac{\partial \mathbf{v}_{\mathbf{k}}}{\partial t}+\frac{\theta_{0}}{\lambda_{a} M^{2}}\left(-i \mathbf{k} \pi_{\mathbf{k}}+\frac{\partial \pi_{\mathbf{k}}}{\partial z} \delta_{i 3}\right)+\frac{1}{\lambda_{a} M^{2}} \frac{\mathrm{d} \pi_{0}}{\mathrm{~d}_{z}} \delta_{i 3} \theta_{\mathbf{k}}= \\
& =-\frac{1}{2 \pi} \int_{-\infty}^{+\infty} \int_{-\infty}^{\infty} \mathbf{k}^{\prime}\left[i\left(\mathbf{k}-\mathbf{k}^{\prime}\right) \cdot \mathbf{u}_{\mathbf{k}^{\prime}}+w_{\mathbf{k}^{\prime}} \frac{\partial}{\partial z}\right] \mathbf{v}_{\mathbf{k}-\mathbf{k}^{\prime}}- \\
& -\frac{1}{2 \pi} \frac{1}{\lambda_{a} M^{2}} \int_{-\infty}^{+\infty} \int_{\mathbf{k}^{\prime}} \theta_{\mathbf{k}^{\prime}}\left[i\left(\mathbf{k}-\mathbf{k}^{\prime}\right) \pi_{\mathbf{k}-\mathbf{k}^{\prime}}+\frac{\partial}{\partial z} \pi_{\mathbf{k}-\mathbf{k}^{\prime}} \delta_{i 3}\right], \\
& \frac{\partial \pi_{\mathbf{k}}}{\partial t}-\pi_{0}(\gamma-1) i \mathbf{k} \cdot \mathbf{u}_{\mathbf{k}}+\left[\frac{\mathrm{d} \pi_{0}}{\mathrm{~d} z}+\pi_{0}(\gamma-1) \frac{\partial}{\partial z}\right] w_{\mathbf{k}}= \\
& =-\frac{1}{2 \pi} \int_{-\infty}^{+\infty} \int_{\mathbf{d} \mathbf{k}^{\prime}}\left[i\left(\mathbf{k}-\mathbf{k}^{\prime}\right) \cdot \mathbf{u}_{\mathbf{k}^{\prime}}+w_{\mathbf{k}^{\prime}} \cdot \frac{\partial}{\partial z}\right] \pi_{\mathbf{k}-\mathbf{k}^{\prime}}- \\
& -\frac{(\gamma-1)}{2 \pi} \int_{-\infty}^{+\infty} \int^{\infty} \mathrm{d} \mathbf{k}^{\prime} \pi_{\mathbf{k}^{\prime}}\left[i\left(\mathbf{k}-\mathbf{k}^{\prime}\right) \cdot \mathbf{u}_{\mathbf{k}-\mathbf{k}^{\prime}}+\frac{\partial}{\partial z} w_{\mathbf{k}-\mathbf{k}^{\prime}}\right], \\
& \frac{\partial \theta_{\mathbf{k}}}{\partial t}+\frac{\mathrm{d} \theta_{0}}{\mathrm{~d} z} w_{\mathbf{k}}=-\frac{1}{2 \pi} \int_{-\infty}^{+\infty} \int_{0}^{\infty} \mathrm{d} \mathbf{k}^{\prime}\left[i\left(\mathbf{k}-\mathbf{k}^{\prime}\right) \cdot \mathbf{u}_{\mathbf{k}^{\prime}}+w_{\mathbf{k}^{\prime}} \frac{\partial}{\partial z}\right] \theta_{\mathbf{k}-\mathbf{k}^{\prime}}
\end{aligned}
$$


Here $\mathbf{k}$ is the horizontal wavenumber vector with components $\left(k_{x}, k_{y}, 0\right) \cdot \mathbf{v}_{\mathbf{k}}, \pi_{\mathbf{k}}$ and $\theta_{\mathbf{k}}$ are the Fourier-transforms of $\mathbf{v}^{\prime}, \pi^{\prime}$, and $\theta^{\prime}, \mathbf{u}_{\mathbf{k}}$ is the Fourier-transform of the horizontal velocity with components $\left(u_{1_{k}}, u_{2_{k}}, 0\right)$, and $w_{\mathbf{k}}$ is the Fourier-transform of the vertical velocity.

\section{The Linear Problem}

\subsection{The general polytropic CASE}

The general problem of waves in a polytrope has been studied by Lamb (1932) in the stable case, by Spiegel and Unno (1962) in the unstable case and has been applied to the solar case by Gough (1978). When one considers the fit showed by Gough (1978) between the polytropic case and the solar case, one is struck by the fact that, at least for the category of modes he considers (high $l$ acoustic modes), the fit is not that bad and that one of the main reasons for such a fit must be the stratified nature of the medium which imposes roughly, its characteristics on the modes. This is another reason to use potential variables which are variables that include physically part of the stratification effects. Some more complete expressions, in the case of a finite polytrope, will be given here along with the values for the $\pi^{\prime}$ and $\theta^{\prime}$ fields, but the usual linear problem will not be detailed. For any variable $f^{\prime}$, a solution of the form

$$
f^{\prime}(x, y, z, t)=F(z) \exp [i(\sigma t-\mathbf{k} \cdot \mathbf{x})]
$$

is considered, where $\sigma$ and $\mathbf{k}$ are the dimensional frequency and horizontal wavenumber vector (of components $k_{x}$ and $k_{y}$ and modulus $k$ ).

A general equation can be written for the divergence of the velocity field,

$$
\chi=\frac{\partial u^{\prime}}{\partial x}+\frac{\partial v^{\prime}}{\partial y}+\frac{\partial w^{\prime}}{\partial z}
$$

or its horizontal Fourier-transform $\chi_{k}$,

$$
\begin{aligned}
& \chi_{\mathbf{k}}=--i \mathbf{k} \cdot \mathbf{u}_{\mathbf{k}}+\frac{\partial w_{\mathbf{k}}}{\partial z} \\
& z \frac{\partial^{2} \chi_{\mathbf{k}}}{\partial z^{2}}+(m+2) \frac{\partial \chi_{\mathbf{k}}}{\partial z}+\left[\frac{m+1}{\gamma} M^{2} \sigma^{2}-k^{2} z-\frac{k^{2}}{M^{2} \sigma^{2}}\left(1-\lambda_{a}\right)\right] \chi_{\mathbf{k}}=0
\end{aligned}
$$

Defining $\psi_{\mathbf{k}}$ such that

$$
\psi_{\mathbf{k}}=e^{k z} \chi_{\mathbf{k}},
$$

one obtains the usual equation (Lamb, 1932)

$$
\zeta \frac{\partial^{2} \psi_{\mathbf{k}}}{\partial \zeta^{2}}+(m+2-\zeta) \frac{\partial \psi_{\mathbf{k}}}{\partial \zeta}-\alpha \psi_{\mathbf{k}}=0
$$


where

and

$$
\zeta=2 k z
$$

$$
\begin{aligned}
& 2 \alpha=-\frac{m+1}{\gamma} \omega^{2}+\left(1-\lambda_{a}\right) \frac{1}{\omega^{2}}+m+2, \\
& \omega^{2}=\frac{M^{2} \sigma^{2}}{k} .
\end{aligned}
$$

Equation (3.8) is the differential equation of confluent hypergeometric functions. Two solutions are $M(\alpha, m+2, \zeta)$ and $\left(1 / \zeta^{m+1}\right) M(\alpha-m-1,-m, \zeta)$, where $M(a, b, \zeta)$ is the confluent hypergeometric function of the first kind, sometimes called Kummer's function, and defined by

$$
M(a, b, \zeta)=\sum_{j=0}^{\infty} \frac{(a)_{j}}{(b)_{j}} \frac{\zeta^{j}}{j !}
$$

where

$$
(a)_{0}=1 \text { and }(a)_{j}=a(a+1) \ldots(a+j-1) .
$$

The two solutions are independent, their Wronskian being

$$
\mathscr{W}[1,2]=-\frac{(m+1) e^{\zeta}}{\zeta^{m+2}}
$$

$\zeta$ varies from $2 k z_{s}$ to $2 k\left(z_{s}+d\right)$ and, in the case of an infinite polytrope, $\zeta$ will vary from 0 to $\infty$. Once $\chi_{\mathbf{k}}$ is known, it is fairly easy to deduce that the vertical velocity $W_{\mathbf{k}}$ is given by (Spiegel and Unno, 1962)

$$
\left(\omega^{4}-1\right) W_{\mathbf{k}}=\frac{\gamma e^{-\zeta / 2}}{(m+1) k}\left\{\omega^{2}\left[-\zeta \frac{\partial \psi_{\mathbf{k}}}{\partial \zeta}-(m+1) \psi_{\mathbf{k}}\right]+\frac{1}{2} \zeta \psi_{\mathbf{k}}\left(\omega^{2}+1\right)\right\} .
$$

Some manipulations with the confluent hypergeometric functions can be used (see Appendix A) to cast the velocity into the more convenient form

$$
\begin{aligned}
W_{\mathbf{k}}= & -A \frac{\gamma}{2 k} e^{-\zeta / 2}\left[\frac{1}{\omega^{2}+1} M(\alpha, m+1, \zeta)+\frac{1}{\omega^{2}-1} M(\alpha-1, m+1, \zeta)\right]+ \\
& +B \frac{\gamma}{2 k} \frac{e^{-\zeta / 2}}{m(m+1) \zeta^{m}}\left[\frac{1}{\omega^{2}+1}(\alpha-m-1) M(\alpha-m,-m+1, \zeta)+\right. \\
& \left.+\frac{1}{\omega^{2}-1}(\alpha-1) M(\alpha-m-1,-m+1, \zeta)\right]
\end{aligned}
$$


where $A$ and $B$ are arbitrary constants. Now, if boundary conditions are applied to $W_{\mathbf{k}}$ at the top and the bottom of the layer, a complicated equation determining the eigenvalues $\omega^{2}$ follows.

\subsection{The INFINITE POLYTROPE}

This equation simplifies considerably in the case of an infinite polytrope for which $\zeta$ varies from 0 to $\infty$. We choose the simplest boundary conditions

$$
W_{\mathbf{k}}(0)=W_{\mathbf{k}}(\infty)=0,
$$

and consider from now on only the case of an infinite polytrope. The boundary condition at $\zeta=0$ forces $B=0$ in (3.14) and, using asymptotic expansions for the confluent hypergeometric functions when $\zeta \rightarrow \infty$, Spiegel and Unno (1962) established that the equation for the eigenvalues becomes simply

$$
\alpha=1-n, \quad n=1,2,3 \text {, etc. , }
$$

where $\alpha$ is given by (3.8). One obtains a quadratic equation for $\omega^{2}$ whose solution is (Gough, 1978)

$$
\begin{aligned}
& \omega_{n_{p}}^{2}=\frac{\gamma}{m+1}\left\{n_{p}+\frac{m}{2}+\left[\left(n_{p}+\frac{m}{2}\right)^{2}-\frac{m+1}{\gamma}\left(\lambda_{a}-1\right)\right]^{1 / 2}\right\}, \\
& n_{p}=1,2,3, \text { etc. }, \\
& \omega_{n_{g}}^{2}=\frac{\gamma}{m+1}\left\{n_{g}+\frac{m}{2}-\left[\left(n_{g}+\frac{m}{2}\right)^{2}-\frac{m+1}{\gamma}\left(\lambda_{a}-1\right)\right]^{1 / 2}\right\}, \\
& n_{g}=1,2,3, \text { etc. }
\end{aligned}
$$

From now on, the polytrope is supposed to be unstable, i.e. $\lambda_{a}<1$, which is seen from (2.19) to be the familiar condition of superadiabaticity. The squared frequencies $\omega_{n}^{2}$ are positive and associated with the stable acoustic modes $p_{n}$. The squared frequencies $\omega_{n}^{2}$ are real and negative and associated with the unstable $g_{n}^{-}$modes whose strong interaction will give rise to turbulent convection. As far as the frequencies themselves are concerned, a special notation is required to differentiate the positive and negative parts of each frequency. One defines from (2.9)

$$
\begin{aligned}
& \sigma_{\mathbf{k},+n_{p}}=\frac{\sqrt{k}}{M}\left|\omega_{n_{p}}\right|, \\
& \sigma_{\mathbf{k},-n_{p}}=-\frac{\sqrt{k}}{M}\left|\omega_{n_{p}}\right|, \\
& n_{p}=1,2,3, \text { etc. }
\end{aligned}
$$


Since the $\omega_{n_{g}}^{2}$ are strictly negative, it is convenient to define

and

$$
\bar{\omega}_{n_{g}}^{2}=-\omega_{n_{g}}^{2}
$$

$$
\begin{aligned}
& \sigma_{\mathbf{k},+n_{g}}=\frac{i \sqrt{k}}{M}\left|\bar{\omega}_{n_{g}}\right|, \\
& \sigma_{\mathbf{k},-n_{g}}=-\frac{i \sqrt{k}}{M}\left|\bar{\omega}_{n_{g}}\right|, \\
& n_{g}=1,2,3, \text { etc. }
\end{aligned}
$$

The modes corresponding to (3.21), (3.22), (3.25), and (3.26) will be denoted respectively by $p_{n_{p}}, p_{-n_{p}}, g_{n_{g}}^{-}, g_{-n_{g}}^{-}$. Before going on with the calculation of the eigenfunctions, it is time to mention here that the above procedure has left out two types of modes. These are modes which have zero divergence. The first type has

$$
\omega_{n_{f}}^{2}=1 \text {, }
$$

and is the familiar class of $f$-mode (or Kelvin mode) which will be associated with the index $n_{f}$ where $n_{f}$ is zero symbolically,

$$
\begin{aligned}
& \sigma_{\mathbf{k}, n_{f}}=\frac{\sqrt{k}}{M}, \\
& \sigma_{\mathbf{k},-n_{f}}=-\frac{\sqrt{k}}{M} .
\end{aligned}
$$

The other type has zero frequency and corresponds to the toroïdal modes of the spherical case; and index $n_{t}$ will later be associated with them. They are such that $\omega_{n_{t}}^{2}=0$.

One can now calculate the eigenvectors associated with each type of mode and start with the $p$ and $g$ modes. The calculation is much simplified by the fact that $\alpha$ is a negative integer. In this limit the confluent hypergeometric functions are expressible in terms of generalized Laguerre polynomials,

$$
M(1-n, m+1, \zeta)=\frac{(n-1) !}{(m+1)_{n-1}} L_{n-1}^{m}(\zeta)
$$

where the notation $(m+1)_{n-1}$ is the same as the one used in (3.11). The Laguerre polynomials are given by

$$
L_{n}^{m}(\zeta)=e^{\zeta} \frac{\zeta^{-m}}{n !} \frac{\mathrm{d}^{n}}{\mathrm{~d} \zeta^{n}}\left(e^{-\zeta \zeta^{n+m}}\right)
$$


Using (3.14) with $B=0$ and $A=\omega_{n}^{2}-1$, one gets

$$
W_{\mathbf{k}, j}=-\frac{\gamma}{2 k} e^{-\zeta / 2} Q_{|j|}(\zeta)
$$

where

$$
Q_{|j|}(\zeta)=\frac{|j| !}{(m+1)_{|j|}}\left[L_{|j|}^{m}(\zeta)+\frac{\omega_{|j|}^{2}-1}{\omega_{|, j|}^{2}+1} \frac{m+|j|}{|j|} L_{|j|-1}^{m}(\zeta)\right]
$$

where $j$ stands for $n_{p}, n_{g},-n_{p}$, or $-n_{g}$. From now on $|j|$ will mean that the quantity that depends on $j$ is independent of the sign of the frequency. From (3.5) and the normalization used in (3.33), one gets

$$
\chi_{\mathbf{k}, j}=e^{-\zeta / 2} \frac{(|j|-1) !}{(m+2)_{|j|-1}} L_{|j|-1}^{m+1}(\zeta)
$$

Using (3.33), (3.35), the linear part of Equations (2.26), (2.27), (2.28), and the following identity between generalized Laguerre polynomials:

$$
\zeta L_{n-1}^{m+1}(\zeta)=-\zeta \frac{\mathrm{d}}{\mathrm{d} \zeta} L_{n}^{m}(\zeta)=(n+m) L_{n-1}^{m}(\zeta)-n L_{n}^{m}(\zeta)
$$

it is easy to express the total eigenvector as

$$
\boldsymbol{\varphi}_{\mathbf{k}, j}=\left[\begin{array}{l}
U_{\mathbf{k},|j|} \\
V_{\mathbf{k},|j|} \\
W_{\mathbf{k},|j|} \\
\Pi_{\mathbf{k}, j} \\
-i \frac{k_{y}}{k} \frac{\gamma}{2 k} P_{|j|}(\zeta) \\
\Theta_{\mathbf{k}, j}
\end{array}\right]=e^{-\zeta / 2}\left[\begin{array}{l}
-i \frac{k_{x}}{k} \frac{\gamma}{2 k} P_{|j|}(\zeta) \\
-\frac{\gamma}{2 k} Q_{|j|}(\zeta) \\
-i \frac{M^{2}}{k} \sigma_{\mathbf{k}, j} \frac{(\gamma-1)(m+1)}{(2 k)^{m+1-[(m+1) / \gamma]}} \zeta^{m-[(m+1) / \gamma]} P_{|j|}(\zeta) \\
-\frac{i}{\sigma_{\mathbf{k}, j}} \frac{(m+1-m \gamma)}{(2 k)^{[(m+1) / \gamma]-m}} \zeta^{[(m+1) / \gamma]-m-1} Q_{|j|}(\zeta)
\end{array}\right],
$$

where

$$
P_{|j|}(\zeta)=\frac{|j| !}{(m+1)_{|j|}}\left[L_{|j|}^{m}(\zeta)-\frac{\omega_{|j|}^{2}-1}{\omega_{|j|}^{2}+1} \frac{m+|j|}{|j|} L_{|j|-1}^{m}(\zeta)\right]
$$


and $j$ has the same significance as before. The eigenvector can be expressed with the polynomials of degree $|j|, P_{|j|}$, and $Q_{|j|}$. The use of potential variables gives simple expressions, simply proportional to $P_{|j|}$ and $Q_{|j|}$, for the thermodynamic part of the eigenvector which then looks like the velocity part. This will, later on, ease the task of finding orthogonality relations. The use of any of the original thermodynamic variables would not have had such an effect and would have given a combination of $P_{|j|}$ and $Q_{|j|}$ in the thermodynamic part of the eigenvector. As far as the $f$-modes are concerned, it can be easily verified that they have an analogous expression to (3.37) with $j=0$ if one adopts the conventions

$$
\begin{aligned}
& \omega_{0}^{2}=1, \\
& (m+1)_{0}=1, \\
& {\left[\frac{\omega_{j}^{2}-1}{|j|}\right]_{j=0} L_{-1}^{m}(\zeta)=0 .}
\end{aligned}
$$

One gets the eigenvectors $\varphi_{\mathbf{k}, \pm n_{f}}$ by replacing $j$ by $\pm n_{f}$ in (3.37) and defining

$$
\begin{aligned}
& P_{\left|n_{f}\right|}(\zeta)=P_{0}(\zeta)=L_{0}^{m}(\zeta)=1, \\
& Q_{\left|n_{f}\right|}(\zeta)=Q_{0}(\zeta)=L_{0}^{m}(\zeta)=1 .
\end{aligned}
$$

These modes have zero divergence

$$
\chi_{\mathbf{k}, \pm n_{f}}=0 \text {. }
$$

The toroidal modes can be found directly from the linear part of (2.29), (2.30), and (2.31) and have the following expression:

$$
\varphi_{\mathbf{k}, t}=f(z)\left[\begin{array}{r}
1 \\
-\frac{k_{x}}{k_{y}} \\
0 \\
0 \\
0
\end{array}\right]
$$

where $f(z)$ is an arbitrary function of $z$. Their divergence is also zero:

$$
\chi_{\mathbf{k}, n_{t}}=0 \text {. }
$$

One must now try to build some orthogonality relation between all the modes. One very useful result is the completeness of the system of polynomials $\left\{e^{-\zeta / 2} \zeta^{m / 2} L_{n}^{m}(\zeta)\right\}$, $n=0,1,2$, etc., with respect to square integrable functions on $[0,+\infty]$ (see for example 
Sansone, 1959). This allows us to build at once the following toroidal eigenvectors;

$$
\boldsymbol{\varphi}_{\mathrm{k}, n_{t}}=e^{-\zeta / 2} L_{n_{t}}^{m}(\zeta)\left[\begin{array}{c}
k_{y} \\
-k_{x} \\
0 \\
0 \\
0
\end{array}\right],
$$

where $n_{t}=0,1,2$, etc.

\subsection{Properties of the linear modes}

The first problem that arises in building the non-linear theory with an expansion over the eigenmodes is the completeness problem. When one speaks of completeness properties, one should mention the vector space and the norm with respect to which completeness is defined. In stellar oscillation theory, completeness means, generally, completeness of the operator acting on the Lagrangian displacements $\xi$ (which forms a Hilbert space) with respect to the norm

$$
\left\langle\xi_{1}, \xi_{2}\right\rangle=\int_{0}^{M} \xi_{1} \cdot \xi_{2}^{*} \mathrm{~d} M_{r},
$$

where $\xi_{1}$ and $\xi_{2}$ are arbitrary displacement eigenvectors, $M$ is the mass of the star, $M_{r}$ the mass inside radius $r$, and ${ }^{*}$ is complex conjugation. The results, so far, have been established in the frame of adiabatic theory with 'zero boundary conditions' and use fairly sophisticated techniques of functional analysis. Kaniel and Kovetz (1967) showed the existence of an expansion theorem; Eisenfeld (1969) established completeness in the convectively stable case. More recently, Dyson and Schutz (1979) generalized these results to the case of a differentially rotating body. Their proof appears to us as the most careful and most general one since it includes the convective case. In the non-rotating case the only condition on their completeness result is that the square of the local Brunt-Väisäla frequency has a lower bound (possibly negative!) over the whole star. Some more general results connected with these problems can be found in Eisenfeld (1968) and Weinberger (1968). In the present case, (3.48) is connected with the usual orthogonality relation between the velocity eigenmodes,

$$
\int_{0}^{\infty} \rho_{0} \mathbf{v}_{i} \cdot \mathbf{v}_{k}^{*} \mathrm{~d} z=\delta_{i k},
$$

for a suitable normalization of the eigenvectors, and is due to the orthogonality relation of the generalized Laguerre polynomials

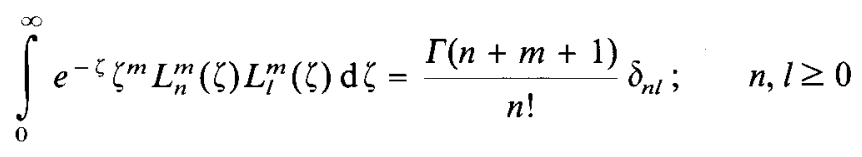


( $\rho_{0}$ is proportional to $\zeta^{m}$ ). Equation (3.50) has the immediate consequence

$$
\begin{aligned}
& \int_{0}^{\infty} \zeta^{m} e^{-\zeta}\left[P_{n}(\zeta) P_{l}(\zeta)+Q_{n}(\zeta) Q_{l}(\zeta)\right] \mathrm{d} \zeta= \\
& =\frac{2 n ! \Gamma(n+m+1)}{\left[(m+1)_{n}\right]^{2}} \delta_{n l}\left[1+\left(\frac{\omega_{n}^{2}-1}{\omega_{n}^{2}+1}\right)^{2} \frac{m+n}{n}\right],
\end{aligned}
$$

where $n$ and $l$ are any of the indices $n_{p}, n_{g}$, and $n_{f}$. (The formula is valid for $n_{f}$ if one follows the convention that $n_{f}$ is associated with the value 0 and $\left[\left(\omega_{\left|n_{f}\right|}^{2}-1\right)^{2}\right] / n_{f}=0$.) Let $\bar{\varphi}$ be the velocity part of the eigenvector $\varphi$, then the following relation ensues from (3.51), (3.17), and (3.19):

$$
\int_{0}^{\infty} \zeta^{m} \bar{\varphi}_{\mathbf{k}, n} \cdot \bar{\varphi}_{\mathbf{k}, l}^{*} \mathrm{~d} \zeta=0 \text { if } n \neq l
$$

where $n$ and $l$ are any of the indices $n_{p}, n_{g}, n_{f}$, or $n_{t}$, and

$$
\int_{0}^{\infty} \zeta^{m}\left|\bar{\varphi}_{\mathbf{k}, n}\right|^{2} \mathrm{~d} \zeta=\frac{\gamma^{2}}{2 k^{2}} \frac{|n| ! \Gamma(|n|+m+1)}{\left[(m+1)_{|n|}\right]^{2}}\left[1+\left(\frac{\omega_{n \mid}^{2}-1}{\omega_{|n|}^{2}+1}\right)^{2} \frac{m+|n|}{|n|}\right],
$$

for $n$ being an index $n_{p}, n_{g}, n_{f}((3.53)$ is the same if one considers the negative frequency modes), and

$$
\int_{0}^{\infty} \zeta^{m}\left|\bar{\varphi}_{\mathbf{k}, n_{t}}\right|^{2} \mathrm{~d} \zeta=k^{2} \frac{\Gamma\left(n_{t}+m+1\right)}{n_{t} !}
$$

for all indices $n_{t}$.

What is needed is a similar orthogonality relation for the eigenvector $\varphi$. The problem is that the completeness result is valid only for the velocity part of the eigenvector $\varphi$ which is insensitive to the sign of the frequency. (Only the thermodynamic part is sensitive to this sign.) Completeness is generally linked with the self-adjointness of the equations, and the equations for the velocity (after elimination of the thermodynamic variables) are self-adjoint with respect to the scalar product (3.49). However, it is fairly easy to prove that for no scalar product of the form

$$
(\varphi, \psi)=\int_{0}^{\infty}\left[z^{m}\left(\varphi_{1} \psi_{1}^{*}+\varphi_{2} \psi_{2}^{*}+\varphi_{3} \psi_{3}^{*}\right)+\alpha_{44} \varphi_{4} \psi_{4}^{*}+\alpha_{55} \varphi_{5} \psi_{5}^{*}\right] \mathrm{d} z,
$$


where $\alpha_{44}$ and $\alpha_{55}$ are arbitrary functions of $z$, is the differential operator $\mathscr{L}(\mathbf{k}, z, \partial / \partial z)$ involved in the linear part of (2.29), (2.30), and (2.31), and which satisfies the eigenvalue equation

$$
\frac{\partial \varphi_{\mathbf{k}}}{\partial t}+\mathscr{L}\left(\mathbf{k}, z, \frac{\partial}{\partial z}\right) \boldsymbol{\varphi}_{\mathbf{k}}=0,
$$

self-adjoint (one can find a bilinear form satisfying (3.55) and making $\mathscr{L}(\mathbf{k}, z, \partial / \partial z)$ self-adjoint in appearance but this bilinear form is not positive definite). One can think of two ways to tackle the problem.

\subsubsection{The Use of New Unknown Variables}

The inspection of the eigenvector (3.37) reveals that in fact each component, when $n$ varies, forms a complete basis in $z$-space. One can then use the scalar product (3.52), (3.53), and (3.54) for the velocity part and associate a variable $A_{\mathbf{k}, n}(t)$ with it and similarly associate variables $B_{\mathbf{k}, n}(t)$, and $C_{\mathbf{k}, n}(t)$ with the pressure and the temperature. One gets the expansions

$$
\begin{aligned}
& \bar{\varphi}_{\mathbf{k}}=\sum_{n} A_{\mathbf{k}, n}(t) \bar{\varphi}_{\mathbf{k}, n}(z), \\
& \bar{\varphi}_{\mathbf{k}, 4}=\sum_{n} B_{\mathbf{k}, n}(t) \varphi_{\mathbf{k}, n, 4}, \\
& \bar{\varphi}_{\mathbf{k}, 5}=\sum_{n} C_{\mathbf{k}, n}(t) \varphi_{\mathbf{k}, n, 5} .
\end{aligned}
$$

This method will certainly provide completeness but it has the drawback of having several variables.

\subsubsection{The Use of the Adjoint Problem}

We choose the following scalar product in the five-dimensional space of the $\varphi$,

with

$$
\begin{aligned}
\left(\varphi_{\mathbf{k}}, \psi_{\mathbf{k}}\right)= & \int_{0}^{\infty}\left[\alpha\left(\varphi_{\mathbf{k}, 1} \psi_{\mathbf{k}, 1}^{*}+\varphi_{\mathbf{k}, 2} \psi_{\mathbf{k}, 2}^{*}+\varphi_{\mathbf{k}, 3} \psi_{\mathbf{k}, 3}^{*}\right)+\right. \\
& \left.+\alpha_{4} \varphi_{\mathbf{k}, 4} \psi_{\mathbf{k}, 4}^{*}+\alpha_{5} \varphi_{\mathbf{k}, 5} \psi_{\mathbf{k}, 5}^{*}\right] \mathrm{d} z,
\end{aligned}
$$

$$
\begin{aligned}
& \alpha=z^{m}, \\
& \alpha_{4}=z^{-m+[(2(m+1)) / \gamma]}, \\
& \alpha_{5}=z^{-[(2(m+1)) / \gamma]+3 m+2} .
\end{aligned}
$$

As already mentioned in (3.56), the eigenvectors are solution of

$$
\frac{\partial \boldsymbol{\varphi}_{\mathbf{k}}}{\partial t}+\mathscr{L}_{\mathbf{k}} \boldsymbol{\varphi}_{\mathbf{k}}=0
$$


where $\mathscr{L}_{\mathbf{k}}$ is the five-dimensional operator

$$
\mathscr{L}_{\mathbf{k}}=\left[\begin{array}{ccccc}
0 & 0 & 0 & -\frac{\theta_{0}}{\lambda_{a} M^{2}} i k_{x} & 0 \\
0 & 0 & 0 & -\frac{\theta_{0}}{\lambda_{a} M^{2}} i k_{y} & 0 \\
0 & 0 & 0 & \frac{\theta_{0}}{\lambda_{a} M^{2}} \frac{\partial}{\partial z} & \frac{1}{\lambda_{a} M^{2}} \frac{\mathrm{d} \pi_{0}}{\mathrm{~d} z} \\
-i k_{x} \pi_{0}(\gamma-1) & -i k_{y} \pi_{0}(\gamma-1) & \frac{\mathrm{d} \pi_{0}}{\mathrm{~d} z}+\pi_{0}(\gamma-1) \frac{\partial}{\partial z} & 0 & 0 \\
0 & 0 & \frac{\mathrm{d} \theta_{0}}{\mathrm{~d} z} & 0 & 0
\end{array}\right] \text {. }
$$

The adjoint operator $\mathscr{L}_{\mathbf{k}}^{\dagger}$ is defined by

$$
\left(\mathscr{L}_{\mathbf{k}} \boldsymbol{\varphi}_{\mathbf{k}}, \psi_{\mathbf{k}}\right)=\left(\varphi_{\mathbf{k}}, \mathscr{L}_{\mathbf{k}}^{\dagger} \psi_{\mathbf{k}}\right)+\left[M\left(\varphi_{\mathbf{k}}, \psi_{\mathrm{k}}\right)\right]_{0}^{\infty},
$$

where $M\left(\varphi_{\mathbf{k}}, \psi_{\mathbf{k}}\right)$ is the bilinear concomitant. The adjoint operator $\mathscr{L}_{\mathbf{k}}^{\dagger}$ is given by

$$
\begin{aligned}
& \mathscr{L}_{\mathbf{k}}^{\dagger}=\left[\begin{array}{ccc}
0 & 0 & 0 \\
0 & 0 & 0 \\
0 & 0 & 0 \\
\frac{\alpha}{\alpha_{4}} \frac{\theta_{0}}{\lambda_{a} M^{2}} i k_{x} & \frac{\alpha}{\alpha_{4}} \frac{\theta_{0}}{\lambda_{a} M^{2}} i k_{y} & -\frac{1}{\alpha_{4} \frac{\lambda_{a} M^{2}}{\frac{d}{\mathrm{~d} z}}\left(\alpha \theta_{0}\right)+\alpha \theta_{0} \frac{\partial}{\partial z}} \\
0 & 0 & \frac{\alpha}{\alpha_{5}} \frac{1}{\lambda_{a} M^{2}} \frac{\mathrm{d} \pi_{0}}{\mathrm{~d} z}
\end{array}\right] \\
& \frac{\alpha_{4}}{\alpha} \pi_{0}(\gamma-1) i k_{x} \\
& \left.\begin{array}{c}
0 \\
0 \\
\frac{\alpha_{5}}{\alpha} \frac{\mathrm{d} \theta_{0}}{\mathrm{~d} z} \\
0 \\
0
\end{array}\right] \\
& \frac{\alpha_{4}}{\alpha} \pi_{0}(\gamma-1) i k_{y} \\
& \frac{\alpha_{4}}{\alpha} \frac{\mathrm{d} \pi_{0}}{\mathrm{~d} z}-\frac{(\gamma-1)}{\alpha}\left[\frac{\mathrm{d}}{\mathrm{d} z}\left(\alpha_{4} \pi_{0}\right)+\alpha_{4} \pi_{0} \frac{\partial}{\partial z}\right] \\
& 0 \\
& 0
\end{aligned}
$$

and the bilinear concomitant by

$$
M\left(\varphi_{\mathbf{k}}, \psi_{\mathbf{k}}\right)=\alpha_{4} \pi_{0}(\gamma-1) \varphi_{\mathbf{k}, 3} \psi_{\mathbf{k}, 4}^{*}+\alpha \frac{\theta_{0}}{\lambda_{a} M^{2}} \varphi_{\mathbf{k}, 4} \psi_{\mathbf{k}, 3}^{*} .
$$


The boundary condition (3.15) makes $\varphi_{\mathbf{k}, 3}$ vanish at $z=0$ and $z=\infty$; the same condition will be imposed on the adjoint eigenvectors, solutions of

$$
\mathscr{L}_{\mathbf{k}}^{\dagger} \varphi_{\mathbf{k}}^{\dagger}=i \sigma_{\mathbf{k}}^{*} \varphi_{\mathbf{k}}^{\dagger},
$$

and, as a result, the bilinear concomitant (3.68) becomes equal to zero.

It is easy now to calculate the eigenvectors of the adjoint operator by introducing solutions of the form (3.1). The calculation is simplified by the fact that the differential system obtained by eliminating the pressure and the temperature is self-adjoint with respect to the eigenvalues $\sigma_{\mathbf{k}, n}^{2}$. As a consequence, one obtains the same equation for the eigenvalues and the same 'velocity part' for the eigenvectors. The only part left to calculate is the adjoint pressure and temperature which are given by (3.67) and (3.69). One gets for the adjoint eigenvectors

$$
\boldsymbol{\varphi}_{\mathbf{k}, j}^{\dagger}=\left[\begin{array}{c}
U_{\mathbf{k},|j|}^{\dagger} \\
V_{\mathbf{k},|j|}^{\dagger} \\
W_{\mathbf{k}, j \mid}^{\dagger} \\
\Pi_{\mathbf{k}, j}^{\dagger} \\
\Theta_{\mathbf{k}, j}^{\dagger}
\end{array}\right]=e^{-\zeta / 2}\left[\begin{array}{l}
-i \frac{k_{x}}{k} \frac{\gamma}{2 k} P_{\mid j}(\zeta) \\
-i \frac{k_{y}}{k} \frac{\gamma}{2 k} P_{\mid j}(\zeta) \\
-i \sigma_{\mathbf{k}, j}^{*} \frac{\gamma}{(\gamma-1) 2 k^{2}} \frac{\zeta^{m-1-[(m+1) / \gamma]}}{(2 k)^{m-1-[(m+1) / \gamma \mid}} P_{|, j|}(\zeta) \\
\frac{i}{\sigma_{\mathbf{k}, j}^{*}} \frac{\gamma}{2 k M^{2}} \frac{\zeta^{-m-2+[(m+1) / \gamma]}}{(2 k)^{-m-2+[(m+1) / \gamma]}} Q_{|, j|}(\zeta)
\end{array}\right] .
$$

The toroidal modes and $f$ modes are the same as in (3.39)-(3.44), and (3.45) (with the same conventions). From (3.64), (3.66), and (3.69), one gets the following properties:

$$
\begin{aligned}
& \mathscr{L}_{\mathbf{k}} \boldsymbol{\varphi}_{\mathbf{k}, j}=-i \sigma_{\mathbf{k}, j} \boldsymbol{\varphi}_{\mathbf{k}, j}, \\
& \mathscr{L}_{\mathbf{k}}^{\dagger} \boldsymbol{\psi}_{\mathbf{k}, l}^{\dagger}=i \sigma_{\mathbf{k}, l}^{*} \psi_{\mathbf{k}, l}^{\dagger},
\end{aligned}
$$

so that, using (3.66), one obtains

$$
\left(\sigma_{\mathbf{k}, j}-\sigma_{\mathbf{k}, l}\right)\left(\varphi_{\mathbf{k}, j}, \psi_{\mathbf{k}, l}^{\dagger}\right)=0,
$$

which implies, when $\sigma_{\mathbf{k}, j}$ and $\sigma_{\mathbf{k}, l}$ are different, the orthogonality property

$$
\left(\varphi_{\mathbf{k}, j}, \psi_{\mathbf{k}, l}^{\dagger}\right)=0 \quad \text { if } \quad \sigma_{\mathbf{k}, j} \neq \sigma_{\mathbf{k}, l} .
$$

Let now the eigenvectors be normalized such that

$$
\left(\boldsymbol{\Phi}_{\mathbf{k}, j}, \boldsymbol{\Psi}_{\mathbf{k}, l}^{\dagger}\right)=\delta_{j l} .
$$


For this, one needs to calculate

$$
\begin{aligned}
\left(\varphi_{\mathbf{k}, j}, \psi_{\mathbf{k}, j}^{\dagger}\right) & =\int_{0}^{\infty} e^{-\zeta}\left\{\frac{\zeta^{m}}{(2 k)^{m}} \frac{\gamma^{2}}{4 k^{2}}\left[\left(P_{|j|}(\zeta)\right)^{2}+\left(Q_{|j|}(\zeta)\right)^{2}\right]+\right. \\
& \left.+\frac{\zeta^{m-1}}{(2 k)^{m}} \frac{\gamma}{2 k^{2}}\left[(m+1) \omega_{|j|}^{2} P_{j \mid}^{2}(\zeta)+\frac{(m \gamma-m-1)}{\omega_{|j|}^{2}} Q_{|j|}^{2}(\zeta)\right]\right\} \frac{\mathrm{d} \zeta}{2 k},
\end{aligned}
$$

if $j$ is any of $n_{p}, n_{g}, n_{f},-n_{p},-n_{g},-n_{f}$. For the toroidal modes, one gets

$$
\left(\boldsymbol{\varphi}_{\mathbf{k}, n_{t}}, \boldsymbol{\Psi}_{\mathbf{k}, n_{t}}^{\dagger}\right)=\int_{0}^{\infty} e^{-\zeta} \frac{\zeta^{m}}{(2 k)^{m}} k^{2}\left[L_{n_{t}}^{m}(\zeta)\right]^{2} \frac{\mathrm{d} \zeta}{2 k}
$$

The calculation of (3.76) is tedious but straightforward; one has to use the dispersion relation (3.16) and the following identities for Laguerre polynomials

$$
L_{j}^{m}(\zeta)=\sum_{h=0}^{j} L_{h}^{m-1}(\zeta)
$$

and

$$
\int_{0}^{\infty} e^{-\zeta \zeta^{m-1}} L_{j}^{m}(\zeta) L_{l}^{m}(\zeta) \mathrm{d} \zeta=\frac{\Gamma[\operatorname{Inf}(j, l)+m+1]}{[\operatorname{Inf}(j, l)] ! m}
$$

One gets

$$
\begin{aligned}
\left(\boldsymbol{\varphi}_{\mathbf{k}, j}, \boldsymbol{\psi}_{\mathbf{k}, j}^{\dagger}\right)= & N(\mathbf{k}, j)=\frac{1}{(2 k)^{m+1}} \frac{\gamma^{2}}{k^{2}} \frac{|j| ! \Gamma(|j|+m+1)}{\left[(m+1)_{|j|}\right]^{2}} \times \\
& \times\left\{\left(1+\frac{|j|}{m}\right)\left[1+\left(\frac{\omega_{|j|}^{2}-1}{\omega_{|j|}^{2}+1}\right)^{2} \frac{m+|j|}{|j|}\right]-\right. \\
& \left.-\left(\frac{\omega_{j \mid}^{2}-1}{\omega_{|j|}^{2}+1}\right)\left[\frac{m+1-m \gamma+(m+1) \omega_{|j|}^{4}}{m \gamma \omega_{|j|}^{2}}\right]\right\},
\end{aligned}
$$

for $j$ belonging to $\pm n_{p}, \pm n_{g}, \pm n_{f}$, and

$$
\left(\varphi_{\mathbf{k}, n_{t}}, \psi_{\mathbf{k}, n_{t}}^{\dagger}\right)=N\left(\mathbf{k}, n_{t}\right)=\frac{1}{(2 k)^{m+1}} \frac{k^{2} \Gamma\left(n_{t}+m+1\right)}{n_{t} !}
$$

This suggests that we define

$$
\boldsymbol{\Phi}_{\mathbf{k}, j}=\varphi_{\mathbf{k}, j}[N(\mathbf{k}, j)]^{-1 / 2}
$$


and

$$
\boldsymbol{\Psi}_{\mathbf{k}, j}^{\dagger}=\boldsymbol{\psi}_{\mathbf{k}, j}[N(\mathbf{k}, j)]^{-1 / 2}
$$

for any index $j$, so that (3.75) is satisfied. (If $N(\mathbf{k}, j)$ is negative, one takes one of the imaginary roots.)

It is now possible to develop on the basis of the eigenvectors $\Phi_{\mathbf{k}, n}$. The general vector $\boldsymbol{\Phi}_{\mathbf{k}}$, solution of (2.29), (2.30), and (2.31), is written

$$
\Phi_{\mathbf{k}}=\sum_{j} A_{\mathbf{k}, j}(t) \Phi_{\mathbf{k}, j}(z)
$$

where, by convection, a summation over index $j$ covers all the indices $\pm n_{p}, \pm n_{g}, \pm n_{f}$, and $n_{t}$. The linear part of the equation, given by (3.64), becomes

$$
\frac{\partial \boldsymbol{\Phi}_{\mathbf{k}}}{\partial t}+\mathscr{L}_{\mathbf{k}} \boldsymbol{\Phi}_{\mathbf{k}}=\sum_{j}\left(\dot{A_{\mathbf{k}, j}}-i \sigma_{\mathbf{k}, j} A_{\mathbf{k}, j}\right) \boldsymbol{\Phi}_{\mathbf{k}, j}(z),
$$

and the equations (2.29), (2.30), and (2.31) are now

$$
\begin{aligned}
& \sum_{j}\left(\dot{A_{\mathbf{k}, j}}-i \sigma_{\mathbf{k}, j} A_{\mathbf{k}, j}\right) \overline{\boldsymbol{\Phi}}_{\mathbf{k}, j}(z)=-\frac{1}{2 \pi} \sum_{j, l} \int_{-\infty}^{+\infty} \int_{-\infty}^{\infty} \mathbf{d k}^{\prime}\left\{\left[i\left(\mathbf{k}-\mathbf{k}^{\prime}\right) \cdot \hat{\boldsymbol{\Phi}}_{\mathbf{k}^{\prime}, j}+\right.\right. \\
& \left.\left.+\Phi_{\mathbf{k}^{\prime}, j, 3} \frac{\partial}{\partial z}\right] \overline{\boldsymbol{\Phi}}_{\mathbf{k}-\mathbf{k}^{\prime}, l}\right\} A_{\mathbf{k}^{\prime}, j} A_{\mathbf{k}-\mathbf{k}^{\prime}, l}-\frac{1}{2 \pi} \frac{1}{\lambda_{a} M^{2}} \sum_{j, l} \int_{-\infty}^{+\infty} \int_{-\infty}^{\infty} \mathrm{k}^{\prime} \times \\
& \times\left\{\Phi_{\mathbf{k}^{\prime}, j, 5}\left[i\left(\mathbf{k}-\mathbf{k}^{\prime}\right) \Phi_{\mathbf{k}-\mathbf{k}^{\prime}, l, 4}+\frac{\partial}{\partial z} \Phi_{\mathbf{k}-\mathbf{k}^{\prime}, l, 4} \delta_{i 3}\right]\right\} A_{\mathbf{k}^{\prime}, j} A_{\mathbf{k}-\mathbf{k}^{\prime}, l} \\
& \sum_{j}\left(\dot{A_{\mathbf{k}, j}}-i \sigma_{\mathbf{k}, j} A_{\mathbf{k}, j}\right) \Phi_{\mathbf{k}, j, 4}=-\frac{1}{2 \pi} \sum_{j, l} \int_{-\infty}^{+\infty} \int_{-\infty}^{\infty} \mathrm{d}^{\prime}\left\{\left[i\left(\mathbf{k}-\mathbf{k}^{\prime}\right) \cdot \hat{\boldsymbol{\Phi}}_{\mathbf{k}^{\prime}, j}+\right.\right. \\
& \left.\left.+\Phi_{\mathbf{k}^{\prime}, j, 3} \frac{\partial}{\partial z}\right] \Phi_{\mathbf{k}-\mathbf{k}^{\prime}, l, 4}\right\} A_{\mathbf{k}^{\prime}, j} A_{\mathbf{k}-\mathbf{k}^{\prime}, l}-\frac{(\gamma-1)}{2 \pi} \sum_{j, l} \int_{-\infty}^{+\infty} \int_{-\infty}^{\infty} \mathrm{d}^{\prime}\left\{\Phi_{\mathbf{k}^{\prime}, j, 4} \times\right. \\
& \left.\times\left[i\left(\mathbf{k}-\mathbf{k}^{\prime}\right) \cdot \hat{\boldsymbol{\Phi}}_{\mathbf{k}-\mathbf{k}^{\prime}, l}+\frac{\mathrm{d}}{\mathrm{d} z} \Phi_{\mathbf{k}-\mathbf{k}^{\prime}, l, 3}\right]\right\} A_{\mathbf{k}^{\prime}, j} A_{\mathbf{k}-\mathbf{k}^{\prime}, l},
\end{aligned}
$$




$$
\begin{aligned}
& \sum_{j}\left(\dot{A_{\mathbf{k}, j}}-i \sigma_{\mathbf{k}, j} A_{\mathbf{k}, j}\right) \Phi_{\mathbf{k}, j, 5}=-\frac{1}{2 \pi} \sum_{j, l} \int_{-\infty}^{+\infty} \int_{-\infty}^{\infty} \mathrm{d}^{\prime} \times \\
& \quad \times\left\{\left[i\left(\mathbf{k}-\mathbf{k}^{\prime}\right) \cdot \boldsymbol{\Phi}_{\mathbf{k}^{\prime}, j}+\Phi_{\mathbf{k}^{\prime}, j, 3} \frac{\mathrm{d}}{\mathrm{d} z}\right] \Phi_{\mathbf{k}-\mathbf{k}^{\prime}, l, 5}\right\} A_{\mathbf{k}^{\prime}, j} A_{\mathbf{k}-\mathbf{k}^{\prime}, l},
\end{aligned}
$$

where $\overline{\boldsymbol{\Phi}}_{\mathbf{k}, j}$ is the velocity part of the eigenvector and $\hat{\boldsymbol{\Phi}}_{\mathbf{k}, j}$ the horizontal velocity vector. The evolution equation of the amplitude of the mode $n$ follows by taking the outer product with $\boldsymbol{\Psi}_{\mathbf{k}, n}^{\dagger}$,

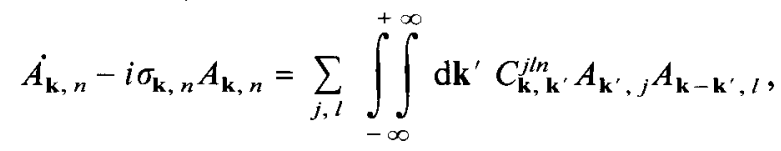

where $C_{\mathbf{k}, \mathbf{k}^{\prime}}^{j / n}$ are the coupling coefficients given in Appendix B. In general, in the development (3.84), the $A_{\mathrm{k}, j}(t)$ are complex coefficients. If one is assured of completeness, the system of Equations (3.89) for all $\mathbf{k}$ and $n$ is equivalent to the initial non-linear equations. Given the completeness result about the velocity vector, completeness in the present case seems to depend only on the fact that, in the linear problem, the relations which link $\Pi_{\mathbf{k}, n}$ and $\Theta_{\mathbf{k}, n}$ to the velocity have coefficients which do not cancel and it seems to be the case here.

\section{The Non-Linear Problem}

At this point, the modes must be classified according to the criterion of Section 1 . So the $f$ and $p$ modes (respectively $g$ and toroïdal) will be considered as pulsating (resp. convective) modes and are associated symbolically with the index $p$ (resp. $c$ ). The equations for both kinds of modes become

$$
\begin{aligned}
\frac{\mathrm{d}}{\mathrm{d} t} A_{\mathbf{k}, p}-i \sigma_{\mathbf{k}, p} A_{\mathbf{k}, p}= & \sum_{c_{1}, c_{2}} \int_{-\infty}^{+\infty} \int_{-\infty}^{\infty} \mathbf{k}^{\prime} C_{\mathbf{k}, \mathbf{k}^{\prime}}^{c_{1} c_{2} p} A_{\mathbf{k}^{\prime}, c_{1}} A_{\mathbf{k}-\mathbf{k}^{\prime}, c_{2}}+ \\
& +\sum_{c_{1}, p_{2}} \int_{-\infty}^{+\infty} \int_{p_{1}, c_{2}} \int_{-\infty}^{\infty} \int^{+} C_{\mathbf{k}, \mathbf{k}^{\prime}}^{c_{1} p_{2} p} A_{\mathbf{k}^{\prime}, c_{1}} A_{\mathbf{k}-\mathbf{k}^{\prime}, p_{2}}+ \\
& +\sum_{\mathbf{k}^{\prime} C_{\mathbf{k}, \mathbf{k}^{\prime}}^{p_{1} c_{2} p} A_{\mathbf{k}^{\prime}, p_{1}} A_{\mathbf{k}-\mathbf{k}^{\prime}, c_{2}}+}^{+\infty} \int_{-\infty}^{+\infty} \int^{+\infty} \mathrm{d} \mathbf{k}^{\prime} C_{\mathbf{k}, \mathbf{k}^{\prime}}^{p_{1} p_{2} p} A_{\mathbf{k}^{\prime}, p_{1}} A_{\mathbf{k}-\mathbf{k}^{\prime}, p_{2}},
\end{aligned}
$$




$$
\begin{aligned}
& \frac{\mathrm{d}}{\mathrm{d} t} A_{\mathbf{k}, c}+\tilde{\sigma}_{\mathbf{k}, c} A_{\mathbf{k}, c}=\sum_{c_{1}, c_{2}} \int_{-\infty}^{+\infty} \int_{-\infty}^{\infty} \mathrm{d}^{\prime} C_{\mathbf{k}, \mathbf{k}^{\prime}}^{c_{1} c_{2} c} A_{\mathbf{k}^{\prime}, c_{1}} A_{\mathbf{k}-\mathbf{k}^{\prime}, c_{2}}+ \\
& +\sum_{c_{1}, p_{2}} \int_{-\infty}^{+\infty} \int_{-\infty}^{\infty} \mathrm{d}^{\prime} C_{\mathbf{k}, \mathbf{k}^{\prime}}^{c_{1} p_{2} c} A_{\mathbf{k}^{\prime}, c_{1}} A_{\mathbf{k}-\mathbf{k}^{\prime}, p_{2}}+ \\
& +\sum_{p_{1}, c_{2}} \int_{-\infty}^{+\infty} \int_{-\infty}^{\infty} \mathbf{k}^{\prime} C_{\mathbf{k}, \mathbf{k}^{\prime}}^{p_{1} c_{2} c} A_{\mathbf{k}^{\prime}, p_{1}} A_{\mathbf{k}-\mathbf{k}^{\prime}, c_{2}}+ \\
& +\sum_{p_{1}, p_{2}} \int_{-\infty}^{+\infty} \int_{-\infty}^{\infty} \mathrm{d} \mathbf{k}^{\prime} C_{\mathbf{k}, \mathbf{k}^{\prime}}^{p_{1} p_{2} c} A_{\mathbf{k}^{\prime}, p_{1}} A_{\mathbf{k}-\mathbf{k}^{\prime}, p_{2}} .
\end{aligned}
$$

In these equations, indices $c, c_{1}$, and $c_{2}$ (resp. $p, p_{1}$, and $p_{2}$ ) are associated with convective (resp. pulsating) modes. $\sigma_{\mathbf{k}, p}$ is real and $\sigma_{\mathbf{k}, c}$ is the imaginary part of $\sigma_{\mathbf{k}, n_{g}}$ or $\sigma_{\mathbf{k},-n_{g}}$ (it is zero for toroïdal modes). All the different-types of coupling have been shown and, as far as interpretation goes, (4.1) is the system of non-linear equations that governs the pulsation. The terms on the right-hand side of (4.1) have a clear physical meaning. The first term is the forcing by the convection and it is very much like a 'Lighthill type' forcing term, though Lighthill (1952) did his calculation for a homogeneous case (see also Lighthill, 1959, 1962; Proudman, 1952; and Pierce and Coroniti, 1966; for a possible example of this type of coupling in geophysics). The second and third terms will have a net damping effect on the pulsation (positive or negative!) and the last term is the wave-wave interaction which gives rise to wave-turbulence.

To simplify these equations, one must exploit the fact that the pulsation is 'almost linear'. This means that we shall try to deal with the pulsation problem as with a wave turbulence problem (i.e. a weakly non linear problem) whereas the convection will stay a fully non-linear problem. Wave-turbulence problems are a familiar class of problems in water-wave theory and in plasma physics. The general problem of weak interaction of surface waves has been studied by Benney and Saffman (1966), Hasselman (1966, 1968), and reviewed by Phillips (1981).

In plasma physics, the evolution of wave correlations has been studied by Davidson (1967). One of the fundamental differences between these cases and the solar case is the inhomogeneity: in general, the wave problems are homogeneous and the linear properties of the problem are simpler than in Section 3. The other difference is that turbulence, when introduced, is generally considered as an external source (in the problem of wind-driven surface waves for instance) and is never perturbed by the waves. In the solar case, it is the goal of the theory to perturb the convection with the oscillations; as a result one has to expect a much more complicated problem. 
Some conditions on $A_{\mathbf{k}, j}$ come from the fact that the vector

$$
\boldsymbol{\Phi}=\left[\begin{array}{c}
u^{\prime} \\
v^{\prime} \\
w^{\prime} \\
\pi^{\prime} \\
\theta^{\prime}
\end{array}\right]=\frac{1}{2 \pi} \int_{-\infty}^{+\infty} \boldsymbol{\Phi}_{\mathbf{k}}(z, t) e^{i \mathbf{k} \cdot \mathbf{x}} \mathrm{d} \mathbf{k}=\frac{1}{2 \pi} \sum_{j} \int_{-\infty}^{+\infty} \int_{\mathbf{k}, j}(t) \Phi_{\mathbf{k}, j}(z) e^{i \mathbf{k} \cdot \mathbf{x}} \mathrm{d} \mathbf{k}
$$

is real. These are

$$
\begin{aligned}
& \text { for } p \text { and } f \text { modes: } A_{-\mathbf{k},-j}=A_{\mathbf{k}, j}^{*}, \\
& \text { for } g \text { and toroidal modes: } A_{-\mathbf{k}, j}=A_{\mathbf{k}, j}^{*} .
\end{aligned}
$$

There are two ways to treat equations (4.1) and (4.2). Either one chooses to expand first with respect to a small parameter and then to carry out statistical averages to obtain equations for the evolution of the correlations or one does these in reverse order. In both cases, one comes up with the same equations though there is a certain amount of debate in the literature about the domain of validity of the expansions which seems to depend on the path followed. In any case, to obtain a uniform development, one has to use multi-time expansions, i.e. perturb the frequencies of the pulsation modes. Here, the method is basically the same as in wave turbulence theory, except that strongly unstable modes (the amplitudes $A_{\mathbf{k}, c}$ ) are now present and they require special treatment. It will be seen that indeed, contrary to the case of wave turbulence, these convective terms dominate the problem. The way to really deal with the problem here is to compute first the different coupling coefficients: there is an infinity of them and it might look a hopeless task, but it is simplified by the symmetries with respect to the indices that these coefficients have, and the fact that one can apply well established asymptotic formulae for the eigenfrequencies and eigenvectors for certain ranges of indices. The key question of the coupling is the ordering of Equations (4.1) and (4.2) when one develops them with respect to the pulsation amplitude (which is small). One has also to understand why it is small. Only when one has defined the expansion procedure and the scaling will it be possible to interpret the expression 'perturbation of the convection by the pulsation' from the equations and there are a priori several ways to do it. We shall propose one that looks reasonable to us and corresponds to what we think about the physics of the coupling, but others may be imagined. The settlement of this question demands the computation of the respective strengths of the coupling coefficients. We shall not go into the detailed calculation of these coupling coefficients here (it involves rather heavy numerical calculations which are not the object of this paper). The following method of scaling the coupling coefficients is introduced: one introduces $\varepsilon$ (the small bookkeeping parameter of the problem) per index $p$ of the coupling coefficients. 
So one gets

$$
\begin{aligned}
& C_{\mathbf{k}, \mathbf{k}^{\prime}}^{c_{1} c_{2} c}=D_{\mathbf{k}, \mathbf{k}^{\prime}}^{c_{1} c_{2} c}, \\
& C_{\mathbf{k}, \mathbf{k}^{\prime}}^{c_{1} p_{2} c}=\varepsilon D_{\mathbf{k}, \mathbf{k}^{\prime}}^{c_{1} p_{2} c}, \\
& C_{\mathbf{k}, \mathbf{k}^{\prime}}^{p_{1} p_{2} c}=\varepsilon^{2} D_{\mathbf{k}, \mathbf{k}^{\prime}}^{p_{1} p_{2} c}, \\
& C_{\mathbf{k}, \mathbf{k}^{\prime}}^{p_{1} p_{2} p}=\varepsilon^{2} D_{\mathbf{k}, \mathbf{k}^{\prime}}^{p_{1} p_{2} p},
\end{aligned}
$$

and so on ... One looks for a solution of the form

$$
\begin{aligned}
& A_{\mathbf{k}, c}=A_{\mathbf{k}, c}^{(0)}+\varepsilon A_{\mathbf{k}, c}^{(1)}+\varepsilon^{2} A_{\mathbf{k}, c}^{(2)}+\ldots, \\
& A_{\mathbf{k}, p}=A_{\mathbf{k}, p}^{(0)}+\varepsilon A_{\mathbf{k}, p}^{(1)}+\varepsilon^{2} A_{\mathbf{k}, p}^{(2)}+\ldots
\end{aligned}
$$

It is also necessary to introduce a procedure to get rid of resonant terms in the 'oscillatory type equations'. There are several ways to do it. Davidson (1967) uses, for example, a two-timing method. Here the frequencies are perturbed according to a procedure that is akin to the Poincaré-Lindstedt method. The new time $s_{\mathbf{k}, p}$ such that

$$
t=s_{\mathbf{k}, p}\left(1+\varepsilon \sigma_{\mathbf{k}, p}^{(1)}+\varepsilon^{2} \sigma_{\mathbf{k}, p}^{(2)}+\ldots\right)
$$

is introduced. $\sigma_{\mathbf{k}, p}^{(1)}, \sigma_{\mathbf{k}, p}^{(2)}$, etc. are chosen so as to kill the resonances appearing on the right-hand side of Equation (4.1). There are other methods to deal with this problem but they all give essentially the same results: they kill the secular terms so that uniformly valid expansions can be obtained. (4.12) establishes a relation between the new shifted frequencies of the system and the finite amplitudes of the perturbations.

The first equation that appears to order $\varepsilon^{0}$ is

$$
\frac{\mathrm{d}}{\mathrm{d} t} A_{\mathbf{k}, c}^{(0)}+\gamma_{\mathbf{k}, c} A_{\mathbf{k}, c}^{(0)}=\sum_{c_{1}, c_{2}} \int_{-\infty}^{+\infty} \int_{-\infty}^{\infty} \mathrm{d}^{\prime} D_{\mathbf{k}, \mathbf{k}^{\prime}}^{c_{1} c_{2} c} A_{\mathbf{k}^{\prime}, c_{1}}^{(0)} A_{\mathbf{k}-\mathbf{k}^{\prime}, c_{2}}^{(0)} .
$$

This is a strongly non-linear problem and an answer to it necessarily asks for a theory of convection (for instance, some closure scheme on the BBGKY hierarchy of equations for the correlations derived from (IV.13) such as the eddy damped quasi-normal markovian scheme (Orszag, 1970) or the test field model (Kraichnan, 1971, 1972); we shall try closures of this type in a later paper). This problem will be supposed to have been solved; in fact, it is the problem on which one wants to put constraints from pulsation theory. (4.13) describes convection in the unperturbed star. The other equations to order $\varepsilon^{0}$ are

$$
\frac{\mathrm{d}}{\mathrm{d} s_{\mathbf{k}, p}} A_{\mathbf{k}, p}^{(0)}-i \sigma_{\mathbf{k}, p} A_{\mathbf{k}, p}^{(0)}=0
$$

with solutions

$$
A_{\mathbf{k}, p}^{(0)}=\alpha_{\mathbf{k}, p} e^{i \sigma_{\mathbf{k}, p} s_{\mathbf{k}, p}}
$$


To order $\varepsilon$, the following equations are obtained:

$$
\begin{aligned}
& \frac{\mathrm{d}}{\mathrm{d} t} A_{\mathbf{k}, c}^{(1)}+\tilde{\sigma}_{\mathbf{k}, c} A_{\mathbf{k}, c}^{(1),}=\sum_{c_{1}, c_{2}} \int_{-\infty}^{+\infty} \int_{-\infty}^{\infty} \mathrm{d} \mathbf{k}^{\prime} D_{\mathbf{k}, \mathbf{k}^{\prime}}^{c_{1} c_{2},}\left(A_{\mathbf{k}^{\prime}, c_{1}}^{(0)} A_{\mathbf{k}-\mathbf{k}^{\prime}, c_{2}}^{(1)}+A_{\mathbf{k}^{\prime}, c_{1}}^{(1)} A_{\mathbf{k}-\mathbf{k}^{\prime}, c_{2}}^{(0)}\right)+ \\
& +\sum_{c_{1}, p_{2}} \int_{-\infty}^{+\infty} \int_{-\infty}^{\infty} \mathrm{d} \mathbf{k}^{\prime} D_{\mathbf{k}, \mathbf{k}^{\prime}}^{c_{1} p_{2} c}\left(A_{\mathbf{k}^{\prime}, c_{1}}^{(0)} A_{\mathbf{k}-\mathbf{k}^{\prime}, p_{2}}^{(0)}\right)+ \\
& +\sum_{p_{1}, c_{2}} \int_{-\infty}^{+\infty} \int_{-\infty}^{\infty} \mathrm{dk}^{\prime} D_{\mathbf{k}_{1}, \mathbf{k}^{\prime}}^{p_{1} c_{2} c}\left(A_{\mathbf{k}^{\prime}, p_{1}}^{(0)} A_{\mathbf{k}-\mathbf{k}^{\prime}, c_{2}}^{(0)}\right) \\
& \frac{\mathrm{d}}{\mathrm{d} s_{\mathbf{k}, p}} A_{\mathbf{k}, p}^{(1)}-i \sigma_{\mathbf{k}, p} A_{\mathbf{k}, p}^{(1)}=i \sigma_{\mathbf{k}, p} \sigma_{\mathbf{k}, p}^{(1)} A_{\mathbf{k}, p}^{(0)}+ \\
& +\sum_{c_{1}, c_{2}} \int_{-\infty}^{+\infty} \int_{-\infty}^{\infty} \mathrm{d} \mathbf{k}^{\prime} D_{\mathbf{k}, \mathbf{k}^{\prime}}^{c_{1} c_{2} p} A_{\mathbf{k}^{\prime}, c_{1}}^{(0)} A_{\mathbf{k}-\mathbf{k}^{\prime}, c_{2}}^{(0)}
\end{aligned}
$$

Equation (4.17) describes the forcing of the pulsation by the convective terms. $\sigma_{\mathbf{k}, p}^{(1)}$ is chosen in such a way that it kills the resonant part of these convective terms. Equation (4.17) is generally the type of equation treated under various forms in the literature to describe the coupling between convection and pulsation (see Goldreich and Keeley, 1977b). However one can barely speak of 'coupling', since convection acts as a simple forcing term. Equation (4.16) is the equation for the perturbation of the convection. It would be more correct, at this stage, to reason directly on correlations, since perturbing the unstable equation (4.2) is a bit ambiguous. One could suppose that $\left\langle A_{\mathbf{k}^{\prime}, p^{\prime}} A_{\mathbf{k}, p^{\prime}}\right\rangle$ reaches a steady state and then perturb the correlation. But the results, as far as equations and expansions are concerned, remain the same. Equation (4.16) is very different in nature from Equation (4.13) despite the resemblance of the left-hand sides: there is no non-linear term on the right-hand side of (4.16). The first term is linear in the amplitude of the perturbed convection and will act as a damping (positive or negative) while the two remaining terms induce pulsation-convection coupling. Consequently, Equation (4.16) will be easier to deal with than (4.13). To order $\varepsilon^{2}$, one gets the equation

$$
\begin{aligned}
& \frac{\mathrm{d}}{\mathrm{d} s_{\mathbf{k}, p}} A_{\mathbf{k}, p}^{(2)}-i \sigma_{\mathbf{k}, p} A_{\mathbf{k}, p}^{(2)}=i \sigma_{\mathbf{k}, p} \sigma_{\mathbf{k}, p}^{(1)} A_{\mathbf{k}, p}^{(1)}+i \sigma_{\mathbf{k}, p} \sigma_{\mathbf{k}, p}^{(2)} A_{\mathbf{k}, p}^{(0)}+ \\
& \quad+\sum_{c_{1}, c_{2}} \int_{-\infty}^{+\infty} \int_{-\infty}^{\infty} \mathrm{dk}^{\prime} D_{\mathbf{k}, \mathbf{k}^{\prime}}^{c_{1} c_{2} p}\left(A_{\mathbf{k}^{\prime}, c_{1}}^{(0)} A_{\mathbf{k}-\mathbf{k}^{\prime}, c_{2}}^{(1)}+A_{\mathbf{k}^{\prime}, c_{1}}^{(1)} A_{\mathbf{k}-\mathbf{k}^{\prime}, c_{2}}^{(0)}\right)+
\end{aligned}
$$




$$
\begin{aligned}
& +\sum_{c_{1}, p_{2}} \int_{-\infty}^{+\infty} \int_{-\infty}^{\infty} \mathrm{d} \mathbf{k}^{\prime} D_{\mathbf{k}, \mathbf{k}^{\prime}}^{c_{1} p_{2} p} A_{\mathbf{k}^{\prime}, c_{1}}^{(0)} A_{\mathbf{k}-\mathbf{k}^{\prime}, p_{2}}^{(0)}+ \\
& +\sum_{p_{1}, c_{2}} \int_{-\infty}^{+\infty} \int^{\infty} \mathrm{d} \mathbf{k}^{\prime} D_{\mathbf{k}, \mathbf{k}^{\prime}}^{p_{1} c_{2} p} A_{\mathbf{k}^{\prime}, p_{1}}^{(0)} A_{\mathbf{k}-\mathbf{k}^{\prime}, c_{2}}^{(0)} .
\end{aligned}
$$

Equation (4.18) is the equation we really want. To this order, $\sigma_{\mathbf{k}, p}^{(2)}$ is chosen to kill the resonances of the right-hand side of the equation. One can now say that the pulsation and the convection have really perturbed each other and that the resulting effect for the pulsation is Equation (4.18). So, in our opinion, Equations (4.13), (4.14), (4.16), (4.17), and (4.18) are the equations applicable to the solar case. One can see that, to order $\varepsilon^{2}$, with the scaling chosen, there is still no interaction of the pulsation with itself. Equation (4.12), to order $\varepsilon^{2}$, gives the frequency shifts as a function of $\varepsilon$. As already emphasized, one can write equations for the correlations between the different amplitudes and it is these equations that have to be dealt with numerically. But, as far as establishing the equations is concerned, the physical interpretation of the different terms that appear in the expansion is more straightforward if one deals directly with the amplitudes.

\section{Conclusion and Prospects}

The polytropic problem is a much simplified version of the full solar problem. In the solar case, five facts complicate the problem. First one has to use a solar model with a convective zone calculated by mixing length theory as the static state. Second, the linear problem of the calculation of eigenmodes and eigenfrequencies has more complicated boundary conditions: one has to allow for running waves above the cutoff frequency which act as a drain of energy on the system and one generally has to put a model atmosphere on top of the solar model to have realistic boundary conditions. Third, the eigenmodes are more varied; there are also $g^{+}$modes that will be put into the wave category (according to the criterion of Section 1). Fourth, some overstable pulsations can be found, for $p$-modes for instance, due to $\kappa$-mechanism (Ando and Osaki, 1975). Finally one has to operate in spherical geometry. None of these facts makes it more difficult, in theory, to deal with the problem along the lines of Sections 3 and 4 . In the problem of non-radial pulsation, perturbation of the convective flux has generally been ignored. So one would use as an eigenbasis the eigenvectors and eigenvalues calculated without coupling and combine them in the non-linear calculation of Section 4, where the perturbation of the convective flux effectively appears. From a numerical point of view, the eigenvectors and eigenfunctions can be calculated one by one for low $l$ and one can use the various asymptotic formulae known for the others. Two cases must be considered to treat the problem of overstability. If it is a pulsation mode which is overstable, then one can scale the unstable part by an $\varepsilon$ and have it interact in the next order, as in the usual wave-turbulence problem (the system is weakly non-linear). If it is a convective mode that is overstable, one can keep convective 
frequencies with imaginary and real parts in the zeroth order problem of the convection and expand on the convective modes with these frequencies.

The procedure of Section 4 links the convection spectrum to the pulsation spectrum. There are three ways to deal with it. First one can try to keep only a few significant modes of each type, truncate, and study the dynamical system that follows. Second, one can try arbitrary theories (mixing length or more recent closures) on Equation (4.13) to see the consequences on the pulsation modes. Third, one can try to use all the dynamical information measured so far on the modes (see Grec et al., 1983; Bos and Hill, 1983) and the more complete information that will be provided by DISCO (see Bonnet, 1983) to derive constraints on the convection spectrum in the solar case. It is a complicated inverse scattering problem, but, as mentioned earlier, this would be the way to understand solar convection a bit better. Moreover more constraints could be derived by applying the same method to the erratically varying white dwarfs that have recently been observed. One can note that the convective equations can be simplified further by differentiating between the equations where $\tilde{\sigma}_{\mathbf{k}, c}>0$ and those where $\tilde{\sigma}_{\mathbf{k}, c}<0$. In the first case, the mode is strongly damped and one can put it equal to zero. These modes can be eliminated and this brings in new coupling coefficients for the modes with $\sigma_{\mathbf{k}, \mathbf{c}}<0$. What we have in mind is that the interaction of these remaining modes produces a spectrum that is perhaps analogous to what is found in the ocean: broad peaks (corresponding to the different cells) superposed on a continuum.

In conclusion, the nature of the non-linear problem in Section 4 should be emphasized. It is homogeneous in $\mathbf{k}$ (i.e. horizontally) and the inhomogeneity appears through the coupling coefficients. Because of this very inhomogeneity, it might turn out that the turbulence problem has simpler features than the usual homogeneous turbulence problems because the coupling coefficients will be imposed roughly by the stratification and one has to expect strong selection rules coming from this. The energy transfers might then be markedly different due to the compressible character of the problem.

\section{Acknowledgements}

I wish to thank D. O. Gough especially, for his encouragements and advice and an anonymous referee for his helpful comments. Moreover I am indebted to the French 'Ministère des Relations Extérieures' for financing my Crimean trip, the Observatoire de Toulouse et Pic-du-Midi for financial support and the director of the Institut d'Astrophysique de Paris, Jean Audouze, for receiving me in his Institute while doing this research.

\section{Appendix A}

The two following relations concerning confluent hypergeometric functions will be useful in what follows:

$$
\frac{\mathrm{d}}{\mathrm{d} \zeta} M(a, b, \zeta)=\frac{a}{b} M(a+1, b+1, \zeta),
$$




$$
M(a, b, \zeta)=M(a+1, b, \zeta)-\frac{\zeta}{b} M(a+1, b+1, \zeta) .
$$

From this, it is easy to deduce the identities

$$
\begin{aligned}
& \zeta \frac{\mathrm{d}}{\mathrm{d} \zeta} M(a, b, \zeta)=a M(a+1, b, \zeta)-a M(a, b, \zeta), \\
& \zeta M(a, b, \zeta)=(a-b) M(a-1, b, \zeta)+(b-2 a) M(a, b, \zeta)+a M(a+1, b, \zeta)
\end{aligned}
$$

The term between brackets of Equation (3.13) is now calculated for each of the independent solutions found for $\psi_{\mathbf{k}}$. One starts with $M(\alpha, m+2, \zeta)$ :

$$
\begin{aligned}
H_{1}= & \omega^{2}\left[-\zeta \frac{\partial M(\alpha, m+2, \zeta)}{\partial \zeta}-(m+1) M(\alpha, m+2, \zeta)\right]+ \\
& +\frac{1}{2} \zeta\left(1+\omega^{2}\right) M(\alpha, m+2, \zeta) .
\end{aligned}
$$

Using (A.3) and (A.4) to express the first and third terms, one gets

$$
\begin{aligned}
H_{1}= & \frac{\alpha}{2}\left(1-\omega^{2}\right) M(\alpha+1, m+2, \zeta)+\left[\frac{m}{2}\left(1-\omega^{2}\right)+1-\alpha\right] \times \\
& \times M(\alpha, m+2, \zeta)+\frac{1}{2}\left(1+\omega^{2}\right)(\alpha-m-2) M(\alpha-1, m+2, \zeta) .
\end{aligned}
$$

(A.6) can now be cast into the form

$$
\begin{aligned}
H_{1}= & \frac{1-\omega^{2}}{2}\{\alpha M(\alpha+1, m+2, \zeta)+(m+1-\alpha) M(\alpha, m+2, \zeta)\}+ \\
& +\frac{1+\omega^{2}}{2}\{(1-\alpha) M(\alpha, m+2, \zeta)+(\alpha-m-2) M(\alpha-1, m+2, \zeta)\}
\end{aligned}
$$

Using the identity

$$
(1+a-b) M(a, b, \zeta)-a M(a+1, b, \zeta)+(b-1) M(a, b-1, \zeta)=0,
$$

to express the first and fourth terms in (A.7), (A.7) finally becomes

$$
H_{1}=(m+1)\left\{\frac{1-\omega^{2}}{2} M(\alpha, m+1, \zeta)-\frac{1+\omega^{2}}{2} M(\alpha-1, m+1, \zeta)\right\}
$$


Applying the same kind of transformation to

$$
\begin{aligned}
H_{2}= & \omega^{2}\left\{-\zeta \frac{\partial}{\partial \zeta}\left[\frac{M(\alpha-m-1,-m, \zeta)}{\zeta^{m+1}}\right]-\right. \\
& \left.-\frac{(m+1)}{\zeta^{m+1}} M(\alpha-m-1,-m, \zeta)\right\}+ \\
& +\frac{1}{2}\left(1+\omega^{2}\right) \frac{M(\alpha-m-1,-m, \zeta)}{\zeta^{m}},
\end{aligned}
$$

one obtains

$$
H_{2}=\frac{\omega^{2}}{\zeta^{m}} \frac{\partial}{\partial \zeta} M(\alpha-m-1,-m, \zeta)+\frac{1}{2}\left(1+\omega^{2}\right) \frac{M(\alpha-m-1,-m, \zeta)}{\zeta^{m}}
$$

Using (A.1) and (A.8), one obtains the identity

$$
b \frac{\mathrm{d}}{\mathrm{d} \zeta} M(a, b, \zeta)=b M(a, b, \zeta)+(a-b) M(a, b+1, \zeta)
$$

which can be used to transform the first term in (A.11),

$$
\begin{aligned}
H_{2}=\frac{1}{\zeta^{m}} & \left\{\frac{\left(1-\omega^{2}\right)}{2} M(\alpha-m-1,-m, \zeta)+\frac{(\alpha-1) \omega^{2}}{m} \times\right. \\
& \times M(\alpha-m-1,-m+1, \zeta)\} .
\end{aligned}
$$

Using (A.8) to transform the first term, one gets

$$
\begin{aligned}
H_{2}= & \frac{1}{\zeta^{m}}\left\{\frac{1+\omega^{2}}{2} \frac{(\alpha-1)}{m} M(\alpha-m-1,-m+1, \zeta)-\right. \\
& \left.-\left(\frac{1-\omega^{2}}{2}\right) \frac{(\alpha-m-1)}{m} M(\alpha-m,-m+1, \zeta)\right\} .
\end{aligned}
$$

When one regroups $H_{1}$ and $H_{2}$ given by (A.9) and (A.14) with (3.13), one gets (3.14) for the velocity. 


\section{Appendix B}

The expression for the coupling coefficient $C_{\mathbf{k}, \mathbf{k}^{\prime}}^{\text {in }}$ is the following:

$$
\begin{aligned}
& C_{\mathbf{k}, \mathbf{k}^{\prime}}^{j / n}=-\frac{1}{2 \pi} \int_{0}^{\infty} \mathrm{d} z z^{m}\left\{i\left(\mathbf{k}-\mathbf{k}^{\prime}\right) \cdot \hat{\boldsymbol{\Phi}}_{\mathbf{k}^{\prime}, j}\left(\overline{\boldsymbol{\Phi}}_{\mathbf{k}-\mathbf{k}^{\prime}, l} \cdot \overline{\boldsymbol{\Psi}}_{\mathbf{k}, n}^{\dagger}\right)+\right. \\
& \left.+\Phi_{\mathbf{k}^{\prime}, j, 3}\left[\left(\frac{\mathrm{d}}{\mathrm{d} z} \overline{\boldsymbol{\Phi}}_{\mathbf{k}-\mathbf{k}^{\prime}, l}\right) \cdot \overline{\boldsymbol{\Psi}}_{\mathbf{k}, n}^{\dagger}\right]\right\}- \\
& -\frac{1}{\lambda_{a} M^{2}} \frac{1}{2 \pi} \int_{0}^{\infty} \mathrm{d} z z^{m} \Phi_{\mathbf{k}^{\prime}, j, 5}\left[i\left(\mathbf{k}-\mathbf{k}^{\prime}\right) \cdot \hat{\Psi}_{\mathbf{k}, n}^{+} \Phi_{\mathbf{k}-\mathbf{k}^{\prime}, l, 4}+\right. \\
& \left.+\left(\frac{\mathrm{d}}{\mathrm{d} z} \Phi_{\mathbf{k}-\mathbf{k}^{\prime}, 1,4}\right) \Psi_{\mathbf{k}, n, 3}^{\dagger}\right]- \\
& -\frac{1}{2 \pi} \int_{0}^{\infty} \mathrm{d} z z^{-m+[(2(m+1)) / \gamma]}\left[i\left(\mathbf{k}-\mathbf{k}^{\prime}\right) \cdot \hat{\boldsymbol{\Phi}}_{\mathbf{k}^{\prime}, j} \Phi_{\mathbf{k}-\mathbf{k}^{\prime}, l, 4} \Psi_{\mathbf{k}, n, 4}^{\dagger}+\right. \\
& \left.+\Phi_{\mathbf{k}^{\prime}, j, 3}\left(\frac{\mathrm{d}}{\mathrm{d} z} \Phi_{\mathbf{k}-\mathbf{k}^{\prime}, l, 4}\right) \Psi_{\mathbf{k}, n, 4}^{\dagger}\right]- \\
& -\frac{(\gamma-1)}{2 \pi} \int_{0}^{\infty} \mathrm{d} z z^{-m+[(2(m+1)) / \gamma]} \Phi_{\mathbf{k}^{\prime}, j, 4} \Psi_{\mathbf{k}, n, 4}^{\dagger}\left[i\left(\mathbf{k}-\mathbf{k}^{\prime}\right) \cdot \hat{\boldsymbol{\Phi}}_{\mathbf{k}-\mathbf{k}^{\prime}, l}+\right. \\
& \left.+\left(\frac{\mathrm{d}}{\mathrm{d} z} \Phi_{\mathbf{k}-\mathbf{k}^{\prime}, 1,3}\right)\right]- \\
& -\frac{1}{2 \pi} \int_{0}^{\infty} \mathrm{d} z z^{-[(2(m+1)) / \gamma]+3 m+2}\left[i\left(\mathbf{k}-\mathbf{k}^{\prime}\right) \cdot \hat{\boldsymbol{\Phi}}_{\mathbf{k}^{\prime}, j} \Phi_{\mathbf{k}-\mathbf{k}^{\prime}, l, 5} \Psi_{\mathbf{k}, n, 5}^{\dagger}+\right. \\
& \left.+\Phi_{\mathbf{k}^{\prime}, j, 3}\left(\frac{\mathrm{d}}{\mathrm{d} z} \Phi_{\mathbf{k}-\mathbf{k}^{\prime}, l, 5}\right) \Psi_{\mathbf{k}, n, 5}^{\dagger}\right]
\end{aligned}
$$

where the symbols $\hat{\Psi}^{\dagger}$ and $\bar{\Psi}^{\dagger}$ have the same meanings as those introduced for $\boldsymbol{\Phi}$. Ingeneral, performing the integrals in $z$ will give rise to some selection rules between $j$, $l$, and $n$. 


\section{References}

Ando, H. and Osaki, Y.: 1975, Publ. Astron. Soc. Japan 27, 581.

Ando, H. and Osaki, Y.: 1977, Publ. Astron. Soc. Japan 29, 221.

Benney, D. J. and Saffman, P. G.: 1966, Proc. Roy. Soc. London A289, 301.

Bonnet, R. M.: 1983, Solar Phys. 82, 487 (this volume).

Bos, R. J. and Hill, H. A.: 1983, Solar Phys. 82, 89 (this volume).

Christensen-Dalsgaard, J. and Frandsen, S.: 1983, Solar Phys. 82, 165 (this volume).

Davidson, R. C.: 1967, J. Plasma Phys. 1, 341.

Dyson, J. and Schutz, B.: 1979, Proc. Roy. Soc. London A368, 389.

Eisenfeld, J.: 1968, J. Math. Anal. Appl. 23, 58.

Eisenfeld, J.: 1969, J. Math. Anal. Appl. 26, 357.

Goldreich, P. and Keeley, D. A.: 1977a, Astrophys. J. 211, 934.

Goldreich, P. and Keeley, D. A.: 1977b, Astrophys. J. 212, 243.

Gough, D. O.: 1969, J. Atmospheric Sci. 26, 448.

Gough, D. O.: 1976, Astrophys. J. 214, 196.

Gough, D. O.: 1978, in G. Belvedere and L. Paterno (eds.), Proc. Workshop on Solar Rotation, Univ. Catania Press, p. 255.

Gough, D. O. and Weiss, N. O.: 1976, Monthly Notices Roy. Astron. Soc. 176, 589.

Grec, G., Fossat, E., and Pomerantz, M.: 1980, Nature 288, 541.

Grec, G., Fossat, E., and Pomerantz, M. A.: 1983, Solar Phys. 82, 55 (this volume).

Hasselman, K.: 1966, Proc. Roy. Soc. London A289, 77.

Hasselman, K.: 1968, in M. Holt (ed.), Basic Developments in Fluid Dynamics, Vol. 2, Academic Press.

Hurle, D. T. J., Jakeman, E., and Pike, E. R.: 1967, Proc. Roy. Soc. London A296, 469.

Kaniel, S. and Kovetz, A.: 1967, Phys. Fluids 10, 1186.

Kraichnan, R. H.: 1971, J. Fluid Mech. 47, 513.

Kraichnan, R. H.: 1972, J. Fluid Mech. 56, 287.

Lamb, H.: 1932, Hydrodynamics, 6th edition, Dover.

Lighthill, M. J.: 1952, Proc. Roy. Soc. London A211, 564.

Lighthill, M. J.: 1954, Proc. Roy. Soc. London A222, 1.

Lighthill, M. J.: 1962, Proc. Roy. Soc. London A267, 147.

Ogura, Y. and Phillips, N. A.: 1962, J. Atmospheric Sci. 19, 173.

Orszag, S. A.: 1970, J. Fluid. Mech. 41, 363.

Phillips, O. M.: 1981, J. Fluid. Mech. 106, 215.

Pierce, A. D. and Coroniti, S. C.: 1966, Nature 210, 1209.

Poyet, J.-P. and Spiegel, E. A.: 1979, Astron. J. 84, 1918.

Proudman, 1.: 1952, Proc. Roy. Soc. London A214, 119.

Sansone, G.: 1959, Orthogonal Functions, Interscience Publishers, New-York.

Spiegel, E. A. and Unno, W.: 1962, Publ. Astron. Soc. Japan 14, 28.

Unno, W.: 1967, Publ. Astron. Soc. Japan 19, 140.

Unno, W.: 1977, in E. A. Spiegel and J.-P. Zahn (eds.), Problems of Stellar Convection, Springer-Verlag, p. 315.

Unno, W., Osaki, Y., Ando, H., and Shibahashi, H.: 1979, Non-Radial Oscillations of Stars, University of Tokyo Press.

Weinberger, H. F.: 1968, J. Math. Anal. Appl. 21, 506. 